\title{
Analytical Solution of Nonlinear Boundary Value Problem for Fin Efficiency of Convective Straight Fins with Temperature-Dependent Thermal Conductivity
}

\author{
K. Saravanakumar, ${ }^{1}$ V. Ananthaswamy, ${ }^{1}$ M. Subha, ${ }^{2}$ and L. Rajendran ${ }^{1}$ \\ ${ }^{1}$ Department of Mathematics, The Madura College, Madurai 625011, TN, India \\ ${ }^{2}$ Madurai Sivakasi Nadars Pioneer Meenakshi Women's College, Poovanthi 630611, Sivaganga District, TN, India \\ Correspondence should be addressed to L. Rajendran; raj_sms@rediffmail.com
}

Received 9 July 2013; Accepted 2 August 2013

Academic Editors: R. R. Burnette, E. Curotto, and I. Kim

Copyright (c) 2013 K. Saravanakumar et al. This is an open access article distributed under the Creative Commons Attribution License, which permits unrestricted use, distribution, and reproduction in any medium, provided the original work is properly cited.

\begin{abstract}
We have employed homotopy analysis method (HAM) to evaluate the approximate analytical solution of the nonlinear equation arising in the convective straight fins with temperature-dependent thermal conductivity problem. Solutions are presented for the dimensionless temperature distribution and fin efficiency of the nonlinear equation. The analytical results are compared with previous work and satisfactory agreement is noted.
\end{abstract}

\section{Introduction}

The discipline of heat transfer, typically considered an aspect of mechanical engineering and chemical engineering, deals with specific applied methods by which thermal energy in a system is generated, or converted, or transferred to another system. Heat transfer includes the mechanisms of heat conduction, thermal radiation, and mass transfer. The analysis of extended surface heat transfer is extensively presented by Kraus et al. [1]. Arslanturk [2] used decomposition method to evaluate the temperature distribution and analytical expression for the fin efficiency.

In the study of heat transfer, a fin is a surface that extends from an object to increase the rate of heat transfer to or from the environment by increasing convection. Incropera and Dewitt [3] presented a series of studies on the topic of heat transfer. Mokheimer [4] discussed a series of finefficiency curves for annular fins of rectangular, constant heat flow area, triangular, concave parabolic, and convex parabolic profiles for a wide range of radius ratios, and the dimensionless parameter based on the locally variable heat transfer coefficient. The optimum dimensions of circular fins with variable profile and temperature-dependent thermal conductivity have been obtained by Zubair et al. [5]. A new approach to calculate thermal performance of a singular fin with variable thermal properties has been presented by Kou et al. [6].

Joneidi et al. [7] studied an analytical solution of fin efficiency of convective straight fins with temperaturedependent thermal conductivity by the DTM. In this present paper, we first apply homotopy analysis method to obtain an approximation of analytical expression of fin efficiency of convective straight fins with temperature-dependent thermal conductivity. This problem is compared with Joneidi et al. [7].

\section{Mathematical Formulation of the Boundary Value Problem}

Consider a straight fin with a temperature-dependent thermal conductivity, arbitrary constant cross-sectional area $A_{C}$; perimeter $P$ and length $b$ (see Figure 1). The fin is attached to a base surface of temperature $T_{b}$ and extends into a fluid of temperature $T_{a}$, and its tip is insulated. The one-dimensional energy balance equation is given as follows

$$
A_{C} \frac{d}{d x}\left[k(T) \frac{d T}{d x}\right]-P \hbar\left(T_{b}-T_{a}\right)=0
$$


where $T$ is the temperature, $k(T)$ is the temperaturedependent thermal conductivity of the fin material, $P$ is the fin perimeter, and $\hbar$ is the heat transfer coefficient. The thermal conductivity of the fin material is assumed as follows:

$$
k(T)=k_{a}\left[1+\lambda\left(T-T_{a}\right)\right],
$$

where $k_{a}$ is the thermal conductivity at the ambient fluid temperature of the fin and $\lambda$ is the parameter describing the variation of the thermal conductivity. The following dimensionless parameters are introduced [4]:

$$
\begin{gathered}
\theta=\frac{T-T_{a}}{T_{b}-T_{a}}, \quad \xi=\frac{x}{b}, \quad \beta=\lambda\left(T_{b}-T_{a}\right), \\
\psi=\left(\frac{\hbar P b^{2}}{k_{a} A_{C}}\right)^{1 / 2} .
\end{gathered}
$$

Using the previous dimensionless variables, the dimensionless form of (1) becomes as follows:

$$
\frac{d^{2} \theta}{d \xi^{2}}+\beta \theta \frac{d^{2} \theta}{d \xi^{2}}+\beta\left(\frac{d \theta}{d \xi}\right)^{2}-\psi^{2} \theta=0
$$

where $\theta$ is the dimensionless temperature, $\xi$ is the dimensionless coordinate, $\beta$ is the dimensionless parameter describing thermal conductivity, and $\psi$ is the thermogeometric fin parameter. The boundary conditions are given as follows:

$$
\begin{aligned}
& \frac{d \theta}{d \xi}=0 \quad \text { when } \xi=0, \\
& \theta=1 \quad \text { when } \xi=1 .
\end{aligned}
$$

\section{Fin Efficiency}

The heat transfer rate from the fin is found by using Newton's law of cooling.

Consider

$$
Q=\int_{0}^{b} P\left(T-T_{a}\right) d x
$$

The ratio of the fin heat transfer rate to the heat transfer rate of the fin if the entire fin was at the base temperature is commonly called as the fin efficiency:

$$
\eta=\frac{Q}{Q_{\text {ideal }}}=\frac{\int_{0}^{b} P\left(T-T_{a}\right) d x}{P b\left(T_{b}-T_{a}\right)}=\int_{\xi=0}^{1} \theta(\xi) d \xi
$$

\section{Approximation of Analytical Solution}

4.1. Homotopy Analysis Method (HAM). The homotopy analysis method employs the concept of the homotopy from topology to flexibly generate a convergent series solution for nonlinear systems. The HAM [8-13] was devised by Shijun Liao which is a powerful analytical method for solving nonlinear problems. The greater generality of this method often allows for strong convergence of the solution over larger spacial and parameter domains. This method provides an analytical solution in terms of an infinite power series. However, there is a practical need to evaluate this solution and to obtain numerical values from the infinite power series. In order to investigate the accuracy of the homotopy analysis method (HAM) solution with a finite number of terms, the system of differential equations was solved.

The homotopy analysis method [14] is a good technique comparing to another perturbation method. The HAM gives excellent flexibility in the expression of the solution and how the solution is explicitly obtained. Different from all reported perturbation and nonperturbative techniques, the homotopy analysis method itself provides us with a convenient way to control and adjust the convergence region and rate of approximation series, when necessary. Briefly speaking, the homotopy analysis method has the following advantages: it is valid even if a given nonlinear problem does not contain any small/large parameters at all; it can be employed to efficiently approximate a nonlinear problem by choosing different sets of base functions. In this paper, we employ HAM (see Appendix A) to solve the nonlinear equation.

4.2. Solution of Dimensionless Temperature. Using HAM, we can obtain the dimensionless temperature (see Appendix B) as follows:

$$
\begin{aligned}
\theta(\xi)= & \frac{1}{\cosh (\psi)}-\frac{h(h+2) \beta \cosh (2 \psi)}{3 \cosh ^{3}(\psi)} \\
& +\frac{2 h^{2} \beta^{2} \cosh ^{2}(2 \psi)}{9 \cosh ^{5}(\psi)} \\
& \left.-\frac{3 h^{2} \beta^{2} \cosh (3 \psi)}{16 \cosh ^{4}(\psi)}-\frac{h^{2} \beta^{2} \psi \sinh (\psi)}{12 \cosh ^{4}(\psi)}\right) \cosh (\psi \xi) \\
+ & \left(\frac{h(h+2) \beta}{3 \cosh ^{2}(\psi)}-\frac{2 h^{2} \beta^{2} \cosh ^{2}(2 \psi)}{9 \cosh ^{4}(\psi)}\right) \cosh (2 \psi \xi) \\
+ & \frac{3 h^{2} \beta^{2} \cosh ^{2}(3 \psi \xi)}{16 \cosh ^{3}(\psi)}+\frac{h^{2} \beta^{2} \psi \xi \sinh (\psi \xi)}{12 \cosh ^{3}(\psi)} .
\end{aligned}
$$

When $\beta \rightarrow 0$ from (4), the temperature is as follows:

$$
\theta(\xi)=\frac{\cosh (\psi \xi)}{\cosh (\psi)}
$$

This is the exact solution of (4) when $\beta=0$. 
4.3. Determination of Fin Efficiency. Using (7) and (8) we can obtain the fin efficiency given as follows:

$$
\begin{aligned}
\eta= & \frac{1}{\cosh (\psi)}+\frac{2 h^{2} \beta^{2} \cosh ^{2}(2 \psi)}{9 \cosh ^{5}(\psi)} \\
& -\frac{h(h+1) \beta \cosh (2 \psi)}{3 \cosh ^{3}(\psi)} \\
& \left.-\frac{3 h^{2} \beta^{2} \cosh (3 \psi)}{16 \cosh ^{4}(\psi)}-\frac{h^{2} \beta^{2} \psi \sinh (\psi)}{12 \cosh ^{4}(\psi)}\right) \frac{\sinh (\psi)}{\psi} \\
+ & \frac{h(h+7) \beta \sinh ^{2}(2 \psi)}{6 \psi \cosh ^{2}(\psi)}+\frac{h^{2} \beta^{2} \sinh ^{3}(3 \psi)}{16 \psi \cosh ^{3}(\psi)} \\
+ & \frac{h^{2} \beta^{2}\left(\psi \cosh ^{2}(\psi)-\sinh (\psi)\right)}{12 \psi \cosh ^{3}(\psi)} \\
& -\frac{h \beta \cosh (2 \psi) \sinh (\psi)}{3 \psi \cosh ^{3}(\psi)} \\
& -\frac{h^{2} \beta^{2} \cosh (2 \psi) \sinh (2 \psi)}{9 \psi \cosh ^{4}(\psi)}
\end{aligned}
$$

\section{Comparison with Previous Work (Joneidi et al. [7])}

Joneidi and coworker [7] obtained the closed form of the solution of (1) using DTM (Tables 1 and 2) as follows:

$$
\begin{aligned}
\theta(\xi)= & a+\frac{\psi^{2} a}{2(1+\beta a)} \xi^{2}-\frac{\psi^{4} a(2 \beta a-1)}{24(1+\beta a)^{3}} \xi^{4} \\
& +\frac{\psi^{6} a(2 \beta a-1)(14 \beta a-1)}{720(1+\beta a)^{5}} \xi^{6} \\
& -\frac{\psi^{8} a(2 \beta a-1)\left(25592 \beta^{3} a^{3}-7152 \beta^{2} a^{2}+330 \beta a-1\right)}{40320(1+\beta a)^{7}} \xi^{8}+\cdots
\end{aligned}
$$

The value of $a$ can be obtained from the following equation:

$$
\begin{aligned}
\theta(1)= & a+\frac{\psi^{2} a}{2(1+\beta a)}-\frac{\psi^{4} a(2 \beta a-1)}{24(1+\beta a)^{3}} \\
& +\frac{\psi^{6} a(2 \beta a-1)(14 \beta a-1)}{720(1+\beta a)^{5}} \\
& -\frac{\psi^{8} a(2 \beta a-1)\left(25592 \beta^{3} a^{3}-7152 \beta^{2} a^{2}+330 \beta a-1\right)}{40320(1+\beta a)^{7}} \\
& +\cdots=1 .
\end{aligned}
$$

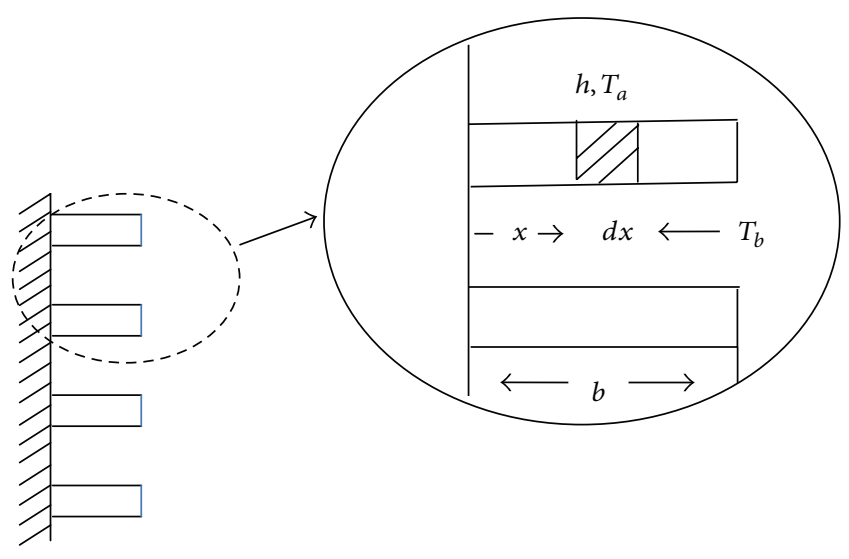

FIGURE 1: The sketch of a straight fin.

The solution of fin efficiency is

$$
\begin{aligned}
\eta= & a+\frac{\psi^{2} a}{6(1+\beta a)}-\frac{\psi^{4} a(2 \beta a-1)}{120(1+\beta a)^{3}} \\
& +\frac{\psi^{6} a(2 \beta a-1)(14 \beta a-1)}{5040(1+\beta a)^{5}} \\
& -\frac{\psi^{8} a(2 \beta a-1)\left(25592 \beta^{3} a^{3}-7152 \beta^{2} a^{2}+330 \beta a-1\right)}{362880(1+\beta a)^{7}} \\
& +\cdots .
\end{aligned}
$$

\section{Discussion}

Equation (8) (this work) and (Equation (11)) (previous work) represent the analytical expressions of temperature for all values of parameters. Among these two expressions our result is the simplest one.

Figure 2 represents dimensionless temperature distribution $\theta$ versus the dimensionless coordinate $\xi$ for various values of $\psi$ using (8). From this figure, it is clear that when $\psi$ increases (i.e., the fin length $b$ increases or the cross-sectional area of the fin $A_{C}$ decreases), the dimensionless temperature $\theta$ decreases.

Figure 3 illustrates the temperature distribution of the fin for different values of $\psi$ and $\beta$. Figure 3(a) depicts the effect of the thermal conductivity $\beta$ on the temperature $\theta$. According to (8) the temperature increases when the thermal conductivity of the fin $\beta$ increases for all values of $\psi$. As an observation, in (10), it can be concluded that the fin efficiency also increases. It is noted that the temperature on fin $\theta$ increases when $\beta$ increases (see Figure 3(b)) for the fixed value of $\psi=1$.

Figure 4 shows the fin efficiency $\eta$ as a function of thermogeometric fin parameter $\psi$ for different values of the thermal conductivity parameter $\beta$ using (10). From this figure, it can be seen that the fin efficiency $\eta$ decreases as $\psi$ increases. However, the fin efficiency $\eta$ increases as the thermal conductivity $\beta$ increases. 
TABLE 1: Our work is compared with Joneidi et al.s [7] work and exact solution of dimensionless temperature $\theta(\xi)$ (linear case).

\begin{tabular}{|c|c|c|c|c|c|c|}
\hline \multirow{2}{*}{$\xi$} & \multicolumn{3}{|c|}{ (a) $\beta=0, \psi=0.5$} & \multicolumn{3}{|c|}{ (b) $\beta=0, \psi=1$} \\
\hline & $\begin{array}{l}\text { HAM (8) } \\
\text { (this work) }\end{array}$ & $\begin{array}{l}\text { DTM [7] } \\
\quad(11)\end{array}$ & Exact & $\begin{array}{l}\text { HAM (8) } \\
\text { (this work) }\end{array}$ & $\begin{array}{c}\text { DTM [7] } \\
\text { (11) }\end{array}$ & Exact \\
\hline 0 & 0.88681 & 0.88681 & 0.88681 & 0.64805 & 0.64805 & 0.64805 \\
\hline 0.05 & 0.88709 & 0.88709 & 0.88709 & 0.64886 & 0.64886 & 0.64886 \\
\hline 0.10 & 0.88792 & 0.88792 & 0.88792 & 0.65129 & 0.65129 & 0.65129 \\
\hline 0.15 & 0.88931 & 0.88931 & 0.88931 & 0.65535 & 0.65535 & 0.65535 \\
\hline 0.20 & 0.89125 & 0.89125 & 0.89125 & 0.66105 & 0.66105 & 0.66105 \\
\hline 0.25 & 0.89375 & 0.89375 & 0.89375 & 0.66841 & 0.66841 & 0.66841 \\
\hline 0.30 & 0.89681 & 0.89681 & 0.89681 & 0.67743 & 0.67743 & 0.67743 \\
\hline 0.35 & 0.90043 & 0.90043 & 0.90043 & 0.68815 & 0.68815 & 0.68815 \\
\hline 0.40 & 0.90461 & 0.90461 & 0.90461 & 0.70059 & 0.70059 & 0.70059 \\
\hline 0.45 & 0.90936 & 0.90936 & 0.90936 & 0.71478 & 0.71478 & 0.71478 \\
\hline 0.50 & 0.91467 & 0.91467 & 0.91467 & 0.73076 & 0.73076 & 0.73076 \\
\hline 0.55 & 0.92056 & 0.92056 & 0.92056 & 0.74856 & 0.74856 & 0.74856 \\
\hline 0.60 & 0.92702 & 0.92702 & 0.92702 & 0.76824 & 0.76824 & 0.76824 \\
\hline 0.65 & 0.93406 & 0.93406 & 0.93406 & 0.78984 & 0.78984 & 0.78984 \\
\hline 0.70 & 0.94169 & 0.94169 & 0.94169 & 0.81341 & 0.81341 & 0.81341 \\
\hline 0.75 & 0.94990 & 0.94990 & 0.94990 & 0.83902 & 0.83902 & 0.83902 \\
\hline 0.80 & 0.95871 & 0.95871 & 0.95871 & 0.86673 & 0.86673 & 0.86673 \\
\hline 0.85 & 0.96812 & 0.96812 & 0.96812 & 0.89660 & 0.89660 & 0.89660 \\
\hline 0.90 & 0.97813 & 0.97813 & 0.97813 & 0.92871 & 0.92871 & 0.92871 \\
\hline 0.95 & 0.98875 & 0.98875 & 0.98875 & 0.96315 & 0.96315 & 0.96315 \\
\hline 1.00 & 1.00000 & 1.00000 & 0.99999 & 1.00000 & 1.00000 & 1.00000 \\
\hline
\end{tabular}

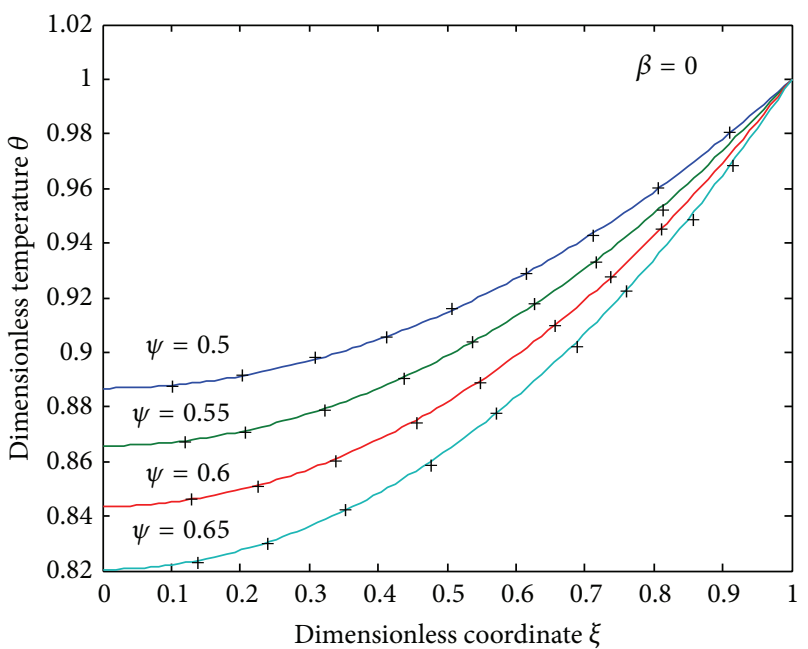

FIGURE 2: Dimensionless temperature $\theta$ versus dimensionless coordinate $\xi$ for various values of $\psi$ when $\beta=0$ and $h=-0.84$. The key to the graph (solid line) represents our approximate solution presented in this work (8), and (+) represents Joneidi et al.s [7] work (Equation (11)).

\section{Conclusions}

There are two main goals that we aimed for this work. The first is to employ the powerful homotopy analysis method to investigate nonlinear differential equation arising in convective straight fins with temperature-dependent thermal conductivity problem. The second is to achieve good results in predicting the solution of the heat transfer equations in engineering. The two goals are achieved successfully. In HAM, we can choose $h$ in an appropriate way which controls the convergence of the series.

\section{Appendices}

\section{A. Basic Idea of Liao's [15] Homotopy Analysis Method}

Consider the following differential equation [15]:

$$
N[u(t)]=0,
$$

where $N$ is a nonlinear operator, $t$ denotes an independent variable, and $u(t)$ is an unknown function. For simplicity, we ignore all boundary or initial conditions, which can be treated in the similar way. By means of generalizing the conventional homotopy method, Liao constructed the so-called zero-order deformation equation as follows:

$$
(1-p) L\left[\varphi(t ; p)-u_{0}(t)\right]=p h H(t) N[\varphi(t ; p)],
$$

where $p \in[0,1]$ is the embedding parameter, $h \neq 0$ is a nonzero auxiliary parameter, $H(t) \neq 0$ is an auxiliary function, $L$ is an auxiliary linear operator, $u_{0}(t)$ is an initial guess 
TABLE 2: Our work is compared with Joneidi et al's [7] work and numerical solution of dimensionless temperature $\theta(\xi)$ (nonlinear case).

\begin{tabular}{|c|c|c|c|c|c|c|}
\hline \multirow{2}{*}{$\xi$} & \multicolumn{3}{|c|}{$\beta=0.4, \psi=1$} & \multicolumn{3}{|c|}{$\beta=0.2, \psi=0.5$} \\
\hline & NS & $\begin{array}{c}\text { DTM [7] } \\
(11)\end{array}$ & $\begin{array}{l}\text { HAM (8) } \\
\text { (this work) }\end{array}$ & NS & $\begin{array}{c}\text { DTM [7] } \\
(11)\end{array}$ & $\begin{array}{l}\text { HAM (8) } \\
\text { (this work) }\end{array}$ \\
\hline 0 & 0.71604 & 0.71604 & 0.71604 & 0.90344 & 0.90344 & 0.90344 \\
\hline 0.05 & 0.71674 & 0.71674 & 0.71674 & 0.90368 & 0.90368 & 0.90368 \\
\hline 0.10 & 0.71883 & 0.71883 & 0.71883 & 0.90440 & 0.90440 & 0.90440 \\
\hline 0.15 & 0.72231 & 0.72231 & 0.72231 & 0.90559 & 0.90559 & 0.90559 \\
\hline 0.20 & 0.72718 & 0.72718 & 0.72718 & 0.90727 & 0.90727 & 0.90727 \\
\hline 0.25 & 0.73346 & 0.73346 & 0.73346 & 0.90942 & 0.90942 & 0.90942 \\
\hline 0.30 & 0.74114 & 0.74114 & 0.74114 & 0.91206 & 0.91206 & 0.91206 \\
\hline 0.35 & 0.75022 & 0.75022 & 0.75022 & 0.91517 & 0.91517 & 0.91517 \\
\hline 0.40 & 0.76072 & 0.76072 & 0.76072 & 0.91877 & 0.91877 & 0.91877 \\
\hline 0.45 & 0.77264 & 0.77264 & 0.77264 & 0.92285 & 0.92285 & 0.92285 \\
\hline 0.50 & 0.78599 & 0.78599 & 0.78599 & 0.92741 & 0.92741 & 0.92741 \\
\hline 0.55 & 0.80077 & 0.80077 & 0.80077 & 0.93246 & 0.93246 & 0.93246 \\
\hline 0.60 & 0.81699 & 0.81699 & 0.81699 & 0.93799 & 0.93799 & 0.93799 \\
\hline 0.65 & 0.83467 & 0.83467 & 0.83467 & 0.94402 & 0.94402 & 0.94402 \\
\hline 0.70 & 0.85381 & 0.85381 & 0.85381 & 0.95053 & 0.95053 & 0.95053 \\
\hline 0.75 & 0.87442 & 0.87442 & 0.87442 & 0.95753 & 0.95753 & 0.95753 \\
\hline 0.80 & 0.89652 & 0.89652 & 0.89652 & 0.96503 & 0.96503 & 0.96503 \\
\hline 0.85 & 0.92011 & 0.92011 & 0.92009 & 0.97302 & 0.97302 & 0.97302 \\
\hline 0.90 & 0.94522 & 0.94522 & 0.94518 & 0.98151 & 0.98151 & 0.98151 \\
\hline 0.95 & 0.97184 & 0.97184 & 0.97181 & 0.99050 & 0.99050 & 0.99050 \\
\hline 1.00 & 1.00000 & 0.99999 & 1.00000 & 0.99999 & 0.99999 & 0.99999 \\
\hline
\end{tabular}

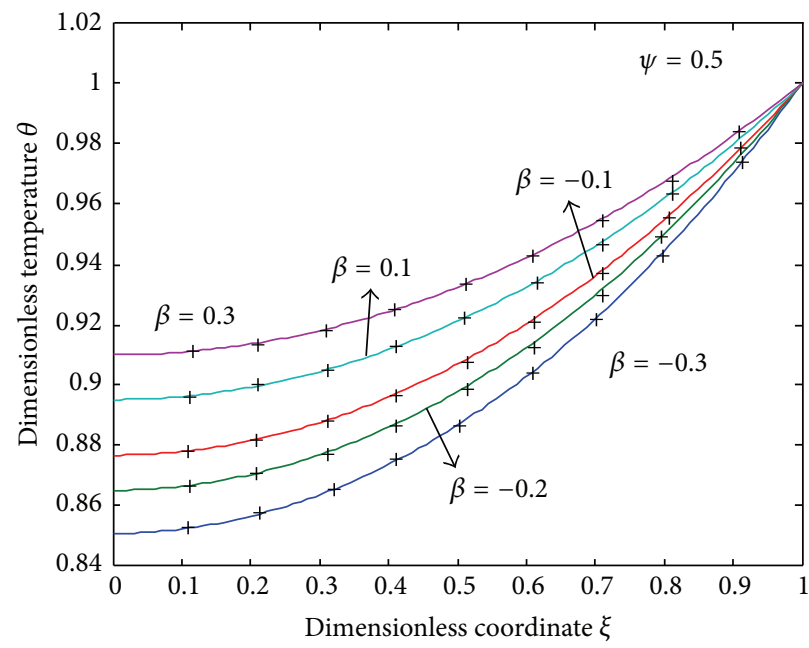

(a)

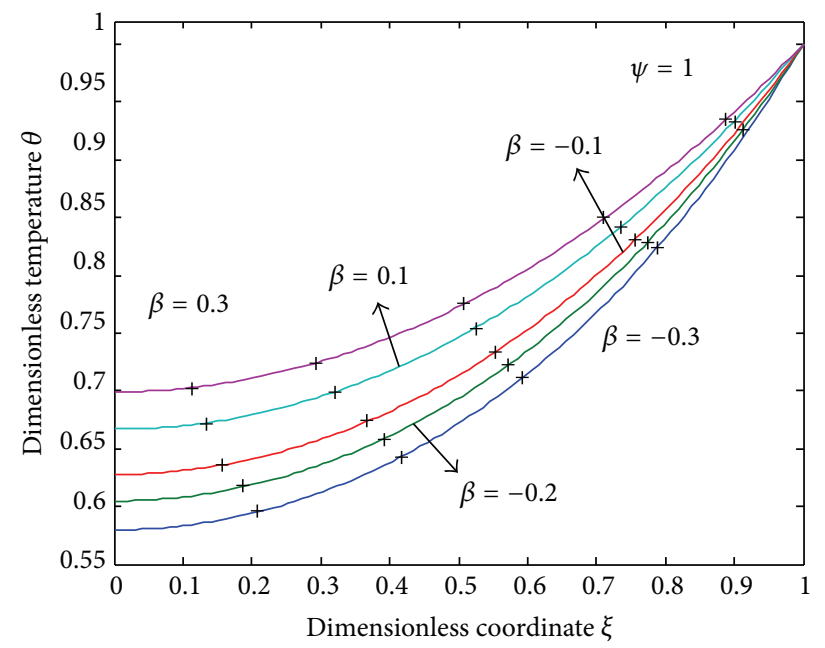

(b)

Figure 3: Dimensionless temperature $\theta$ versus dimensionless coordinate $\xi$ for various values of $\beta$ when (a) $\psi=0.5, h=-0.84$ and (b) $\psi=1, h=-0.84$. The key to the graph (solid line) represents our approximate solution presented in this work (8), and (+) represents Equation (11) [7].

of $u(t)$, and $\varphi(t: p)$ is an unknown function. It is important that one has great freedom to choose auxiliary unknowns in HAM. Obviously, when $p=0$ and $p=1$, it holds:

$$
\varphi(t ; 0)=u_{0}(t), \quad \varphi(t ; 1)=u(t),
$$

respectively. Thus, as $p$ increases from 0 to 1 , the solution $\varphi(t ; p)$ varies from the initial guess $u_{0}(t)$ to the solution $u(t)$. Expanding $\varphi(t ; p)$ in Taylor series with respect to $p$, we have

$$
\varphi(t ; p)=u_{0}(t)+\sum_{m=1}^{+\infty} u_{m}(t) p^{m}
$$




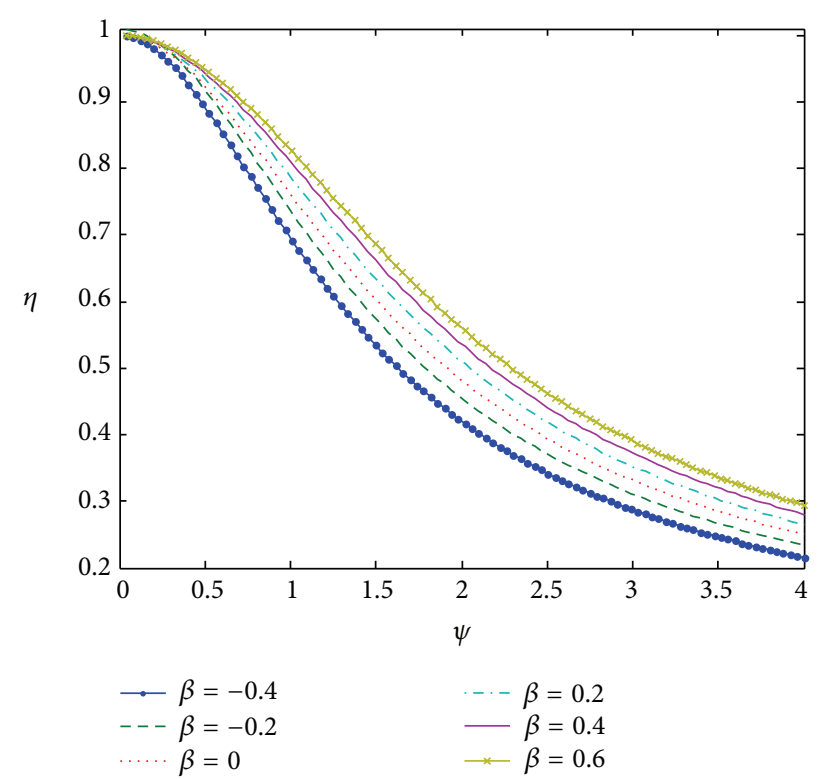

FIGURE 4: Influence of thermal conductivity parameter $\beta$ on the fin efficiency $\eta$ with the thermogeometric fin parameter $\psi$.

where

$$
u_{m}(t)=\left.\frac{1}{m !} \frac{\partial^{m} \varphi(t ; p)}{\partial p^{m}}\right|_{p=0} .
$$

If the auxiliary linear operator, the initial guess, the auxiliary parameter $h$, and the auxiliary function are so properly chosen, the series equation (A.4) converges at $p=1$, then we have

$$
u(t)=u_{0}(t)+\sum_{m=1}^{+\infty} u_{m}(t)
$$

Define the vector

$$
\vec{u}_{n}=\left\{u_{0}, u_{1}, \ldots, u_{n}\right\} .
$$

Differentiating (A.2) for $m$ times with respect to the embedding parameter $p$, then setting $p=0$, and finally dividing them by $m$ !, we will have the so-called $m$ th-order deformation equation as follows:

$$
L\left[u_{m}-\chi_{m} u_{m-1}\right]=h H(t) \Re_{m}\left(\vec{u}_{m-1}\right),
$$

where

$$
\begin{gathered}
\Re_{m}\left(\vec{u}_{m-1}\right)=\left.\frac{1}{(m-1) !} \frac{\partial^{m-1} N[\varphi(t ; p)]}{\partial p^{m-1}}\right|_{p=0}, \\
\chi_{m}= \begin{cases}0, & m \leq 1, \\
1, & m>1 .\end{cases}
\end{gathered}
$$

Applying $L^{-1}$ on both sides of (A.8), we get

$$
u_{m}(t)=\chi_{m} u_{m-1}(t)+h L^{-1}\left[H(t) \Re_{m}\left(\vec{u}_{m-1}\right)\right] .
$$

In this way, it is easily to obtain $u_{m}$ for $m \geq 1$, at $M$ th order, we have

$$
u(t)=\sum_{m=0}^{M} u_{m}(t) .
$$

When $M \rightarrow+\infty$, we get an accurate approximation of the original (A.1). For the convergence of the above method we refer the reader to Liao [15]. If (A.1) admits unique solution, then this method will produce the unique solution. If (A.1) does not possess unique solution, the HAM will give a solution among many other (possible) solutions.

\section{B. Solution of (4) Using Homotopy Analysis Method}

In this appendix, we indicate how (8) in this paper is derived. The homotopy analysis method was constructed to determine the solution of (4)-(5) as follows:

$$
\frac{d^{2} \theta}{d \xi^{2}}+\beta \theta \frac{d^{2} \theta}{d \xi^{2}}+\beta\left(\frac{d \theta}{d \xi}\right)^{2}-\psi^{2} \theta=0 .
$$

In order to solve (B.1) by means of the HAM, we first construct the zeroth-order deformation equation by taking $H(t)=1$.

Consider

$$
\begin{aligned}
& (1-p)\left[\frac{d^{2} \theta}{d \xi^{2}}-\psi^{2} \theta\right] \\
& =p h\left[\frac{d^{2} \theta}{d \xi^{2}}+\beta \theta \frac{d^{2} \theta}{d \xi^{2}}+\beta\left(\frac{d \theta}{d \xi}\right)^{2}-\psi^{2} \theta\right] .
\end{aligned}
$$

The approximate solution of (B.2) is as follows:

$$
\theta=\theta_{0}+p \theta_{1}+p \theta_{2}+\cdots .
$$

Substituting (B.3) into (B.1) and comparing the coefficients of like powers of $p$, we get

$$
\begin{aligned}
& p^{0}: \frac{d^{2} \theta_{0}}{d \xi^{2}}-\psi^{2} \theta_{0}=0, \\
& p^{1}: \frac{d^{2} \theta_{1}}{d \xi^{2}}-\psi^{2} \theta_{1} \\
&=(h+1)\left[\frac{d^{2} \theta_{0}}{d \xi^{2}}-\psi^{2} \theta_{0}\right] \\
&+h \beta\left[\theta_{0} \frac{d^{2} \theta_{0}}{d \xi^{2}}+\left(\frac{d^{2} \theta_{0}}{d \xi^{2}}\right)^{2}\right], \\
& p^{2}: \frac{d^{2} \theta_{2}}{d \xi^{2}}-\psi^{2} \theta_{2} \\
&=(h+1)\left[\frac{d^{2} \theta_{1}}{d \xi^{2}}-\psi^{2} \theta_{1}\right] \\
&+h \beta\left[\theta_{0} \frac{d^{2} \theta_{1}}{d \xi^{2}}+\theta_{1} \frac{d^{2} \theta_{0}}{d \xi^{2}}+2 \frac{d \theta_{0}}{d \xi^{2}} \frac{d \theta_{1}}{d \xi^{2}}\right] .
\end{aligned}
$$




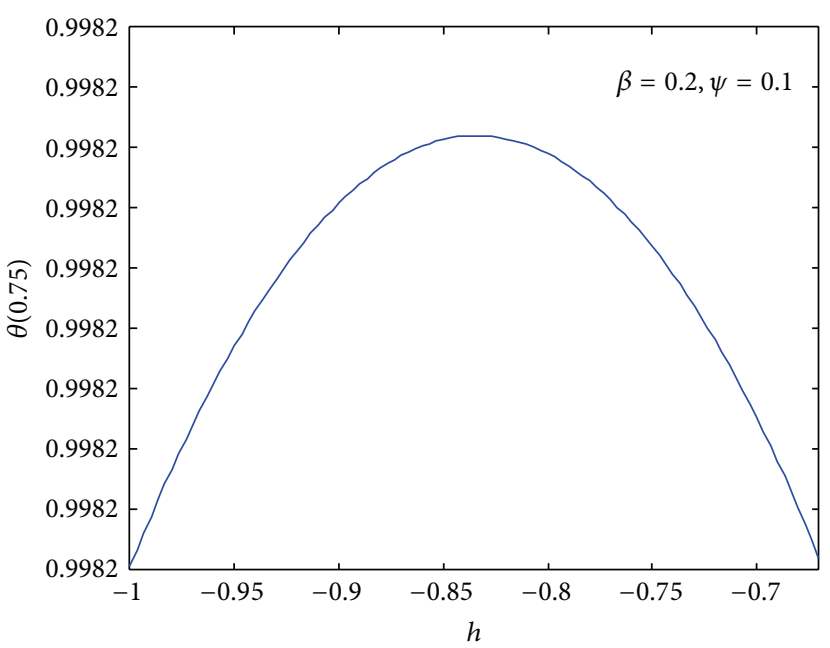

(a)

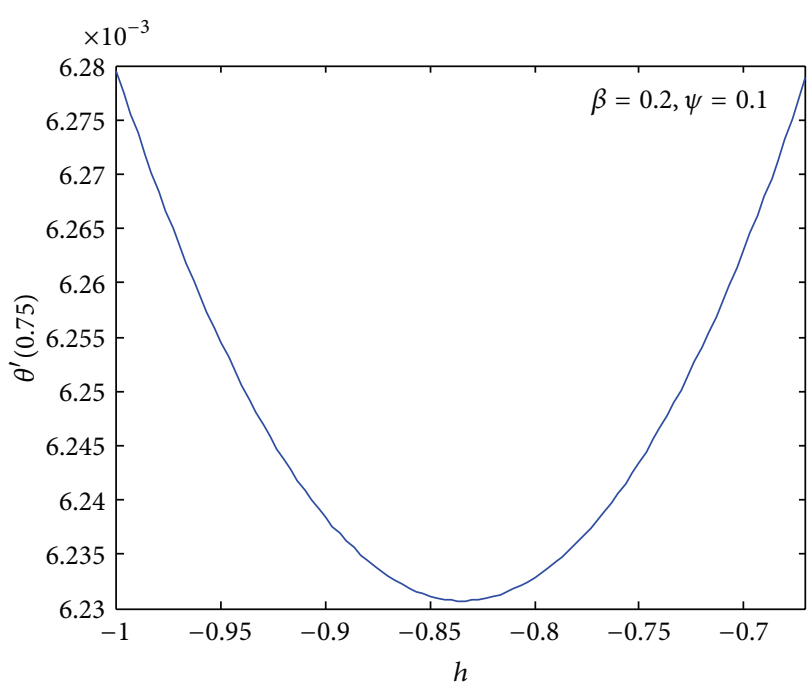

(b)

FIGURE 5: The $h$ curves to indicate the convergence region for $\beta=0.2$ and $\psi=0.1$.

The boundary conditions are

$$
\begin{aligned}
& \frac{d \theta_{0}}{d \xi}=0 \quad \text { when } \xi=0, \quad \theta_{0}=1 \quad \text { when } \xi=1 \text {, } \\
& \frac{d \theta_{i}}{d \xi}=0 \quad \text { when } \xi=0, \quad \theta_{i}=0 \quad \text { when } \xi=1 \\
& \forall i=1,2,3, \ldots
\end{aligned}
$$

Now applying the boundary conditions (B.7) in (B.4) we get

$$
\theta_{0}(\xi)=\frac{\cosh (\psi \xi)}{\cosh (\psi)}
$$

Substituting the value of $\theta_{0}$ in (B.5) and solving the equation using the boundary conditions (B.8), we obtain the following results:

$$
\theta_{1}(\xi)=-\frac{h \beta \cosh (2 \psi)}{3 \cosh ^{3}(\psi)} \cosh (\psi \xi)+\frac{h \beta \cosh (2 \psi \xi)}{3 \cosh ^{2}(\psi)} .
$$

Upon solving (B.6) by substituting the values of $\theta_{0}$ and $\theta_{1}$ and using the boundary conditions equation (B.8), we can find the following results:

$$
\begin{aligned}
\theta_{2}(\xi)= & \frac{2 h^{2} \beta^{2} \cosh ^{2}(2 \psi)}{9 \cosh ^{5}(\psi)}-\frac{h(h+1) \beta \cosh (2 \psi)}{3 \cosh ^{3}(\psi)} \\
& \left.-\frac{3 h^{2} \beta^{2} \cosh (3 \psi)}{16 \cosh ^{4}(\psi)}-\frac{h^{2} \beta^{2} \psi \sinh (\psi)}{12 \cosh ^{4}(\psi)}\right) \cosh (\psi \xi) \\
& +\left(\frac{h(h+1) \beta}{3 \cosh ^{2}(\psi)}-\frac{2 h^{2} \beta^{2} \cosh (2 \psi)}{9 \cosh ^{4}(\psi)}\right) \cosh (2 \psi \xi) \\
& +\frac{3 h^{2} \beta^{2} \cosh ^{2}(3 \psi \xi)}{16 \cosh ^{3}(\psi)}+\frac{h^{2} \beta^{2} \psi \xi \sinh (\psi \xi)}{12 \cosh ^{3}(\psi)} .
\end{aligned}
$$

After three successive iterations the solutions of $\theta$ reach the better approximation. Adding (B.9) to (B.11), we get (8) in the text.

\section{Determining the Region of $h$ for Validity}

The analytical solution should converge. It should be noted that the auxiliary parameter $h$ controls the convergence and accuracy of the solution series. The analytical solution represented by (8) contains the auxiliary parameter $h$, which gives the convergence region and rate of approximation for the homotopy analysis method. In order to define the region such that the solution series is independent of $h$, a multiple of $h$ curves are plotted. The region where the dimensionless temperature $\theta(\xi)$ and $\theta^{\prime}(\xi)$ versus $h$ is a horizontal line known as the convergence region for the corresponding function. The common region among $\theta(\xi)$ and its derivatives are known as the overall convergence region. To study the influence of $h$ on the convergence of solution, $h$-curves of $\theta(0.75)$ and $\theta^{\prime}(0.75)$ are plotted in Figures 5(a) and 5(b), respectively, for $\beta=0.2, \psi=0.1$. These figures clearly indicate that the valid region of $h$ is about -0.86 to -0.82 . Similarly, we can find the value of the convergence control parameter $h$ for different values of the constant parameters.

\section{Nomenclature}

$A_{C}:$ Cross-sectional area of the fin $\left(\mathrm{m}^{2}\right)$

$b$ : $\quad$ Fin length $(\mathrm{m})$

$\hbar$ : Heat transfer coefficient $\left(\mathrm{W} \mathrm{m}^{-1} \mathrm{~K}^{-1}\right)$

$k$ : Thermal conductivity of the fin material (W $\mathrm{m}^{-1} \mathrm{~K}^{-1}$ )

$k_{a}$ : Thermal conductivity at the ambient fluid temperature $\left(\mathrm{W} \mathrm{m}^{-1} \mathrm{~K}^{-1}\right.$ )

$k_{b}$ : Thermal conductivity at the base temperature $\left(\mathrm{W} \mathrm{m}^{-1} \mathrm{~K}^{-1}\right.$ ) 
$P:$ Fin perimeter $(\mathrm{m})$

Q: Heat-transfer rate $(\mathrm{W})$

$T_{a}$ : Temperature of surface $a(\mathrm{~K})$

$T_{b}$ : Temperature of surface $b(\mathrm{~K})$

$x$ : Distance measured from the fin tip (m).

\section{Greek Letters}

$\lambda$ : The slope of the thermal conductivity-temperature curve

$\beta$ : Dimensionless parameter describing variation of the thermal conductivity

$\eta$ : Fin efficiency

$\xi$ : Dimensionless coordinate

$\psi$ : Thermogeometric fin parameter

$\theta$ : Dimensionless temperature.

\section{Acknowledgments}

This work is supported by the University Grant Commission (UGC) Minor project no. F. MRP-4122/12 (MRP/UGCSERO), Hyderabad, Government of India. The authors are thankful to Shri. S. Natanagopal, Secretary at the Madura College Board, and Dr. R. Murali, Principal at the Madura College (Autonomous), Madurai, Tamil Nadu, India, for their constant encouragement.

\section{References}

[1] A. Kraus, A. Aziz, and J. Welty, Extended Surface Heat Transfer, John Wiley \& Sons, New York, NY, USA, 2001.

[2] C. Arslanturk, "A decomposition method for fin efficiency of convective straight fins with temperature-dependent thermal conductivity," International Communications in Heat and Mass Transfer, vol. 32, no. 6, pp. 831-841, 2005.

[3] F. P. Incropera and D. P. Dewitt, Introduction to Heat Transfer, John Wiley \& Sons, New York, NY, USA, 3rd edition, 1996.

[4] E. M. A. Mokheimer, "Performance of annular fins with different profiles subject to variable heat transfer coefficient," International Journal of Heat and Mass Transfer, vol. 45, no. 17, pp. 3631-3642, 2002.

[5] S. M. Zubair, A. Z. Al-Garni, and J. S. Nizami, "The optimal dimensions of circular fins with variable profile and temperature-dependent thermal conductivity," International Journal of Heat and Mass Transfer, vol. 39, no. 16, pp. 3431-3439, 1996.

[6] H.-S. Kou, J.-J. Lee, and C.-Y. Lai, "Thermal analysis of a longitudinal fin with variable thermal properties by recursive formulation," International Journal of Heat and Mass Transfer, vol. 48, no. 11, pp. 2266-2277, 2005.

[7] A. A. Joneidi, D. D. Ganji, and M. Babaelahi, "Differential Transformation Method to determine fin efficiency of convective straight fins with temperature dependent thermal conductivity," International Communications in Heat and Mass Transfer, vol. 36, no. 7, pp. 757-762, 2009.

[8] S. J. Liao, The proposed homotopy analysis technique for the solution of non linear problems [Ph.D. thesis], Shanghai Jiao Tong University, 1992.

[9] S.-J. Liao, "An approximate solution technique not depending on small parameters: a special example," International Journal of Non-Linear Mechanics, vol. 30, no. 3, pp. 371-380, 1995.
[10] S. J. Liao, "On the homotopy analysis method for nonlinear problems," Applied Mathematics and Computation, vol. 147, no. 2, pp. 499-513, 2004.

[11] S. J. Liao, "An optimal homotopy-analysis approach for strongly nonlinear differential equations," Communications in Nonlinear Science and Numerical Simulation, vol. 15, no. 8, pp. 2003-2016, 2010.

[12] S. J. Liao, The Homotopy Analysis Method in Non Linear Differential Equations, Springer, New York, NY, USA, 2012

[13] G. Domairry and H. Bararnia, "An approximation of the analytic solution of some nonlinear heat transfer equations: a survey by using homotopy analysis method," Advanced Studies in Theoretical Physics, vol. 2, no. 11, pp. 507-518, 2008.

[14] H. Jafari, C. Chun, and S. M. Saeidy, "Analytical solution for nonlinear gas dynamic Homotopy analysis method," Applied Mathematics, vol. 4, pp. 149-154, 2009.

[15] S. J. Liao, Beyond Perturbation Introduction to the Homotopy Analysis Method, Chapman \& Hall/CRC, Boca Raton, Fla, USA, 1st edition, 2003. 

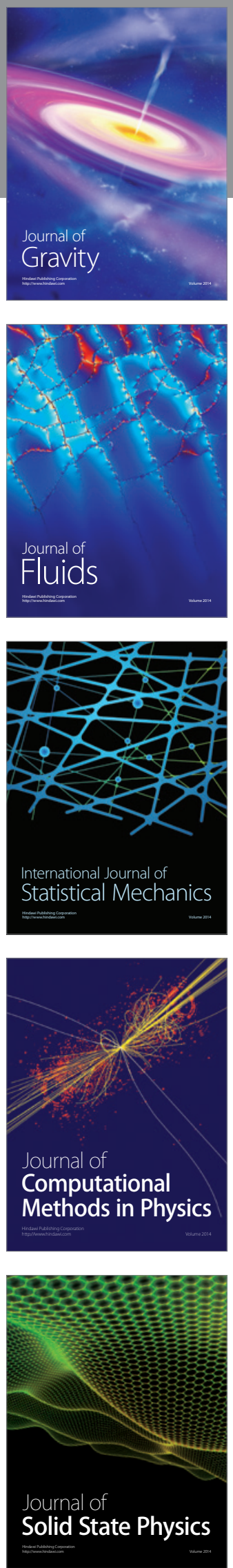

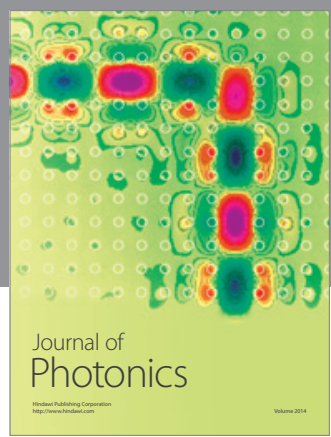

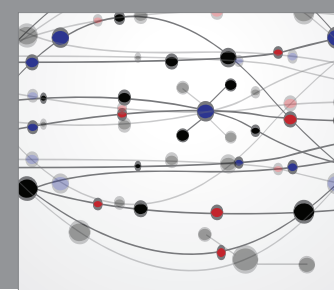

The Scientific World Journal

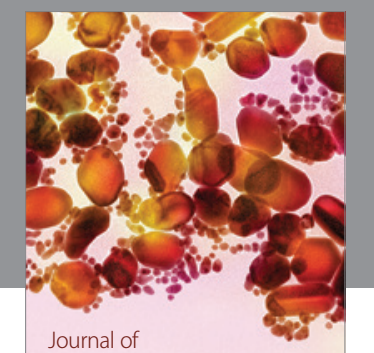

Soft Matter
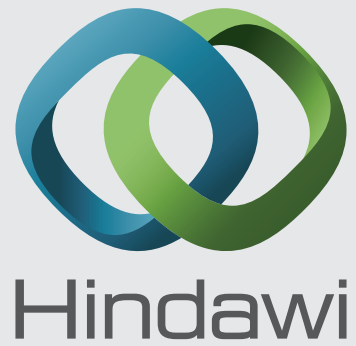

Submit your manuscripts at

http://www.hindawi.com
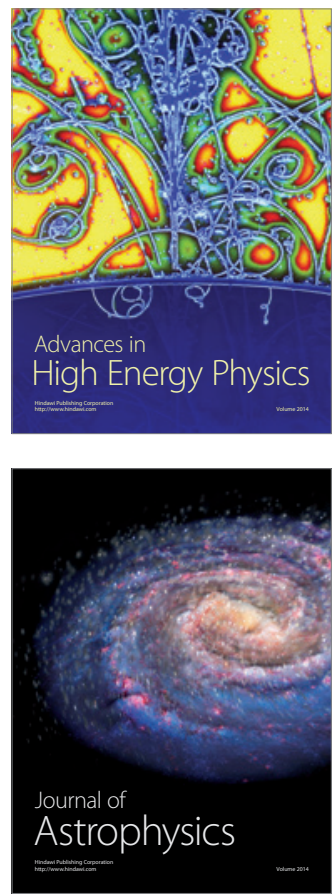
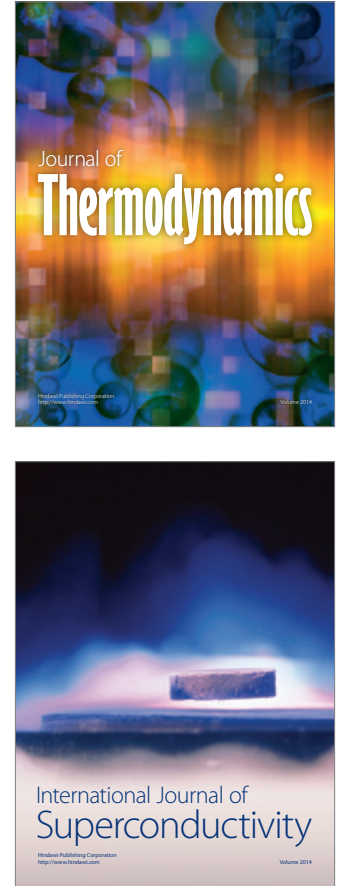
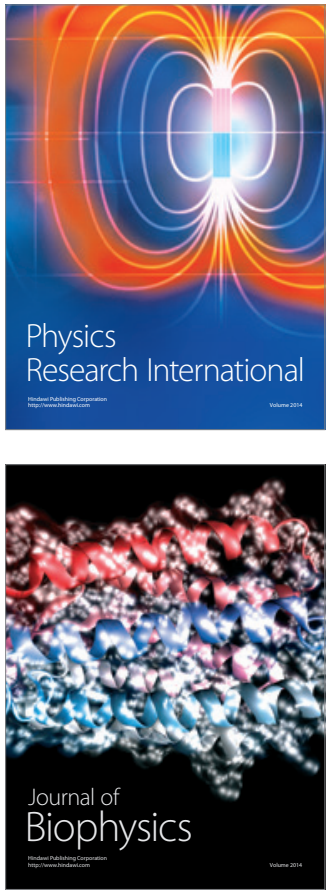
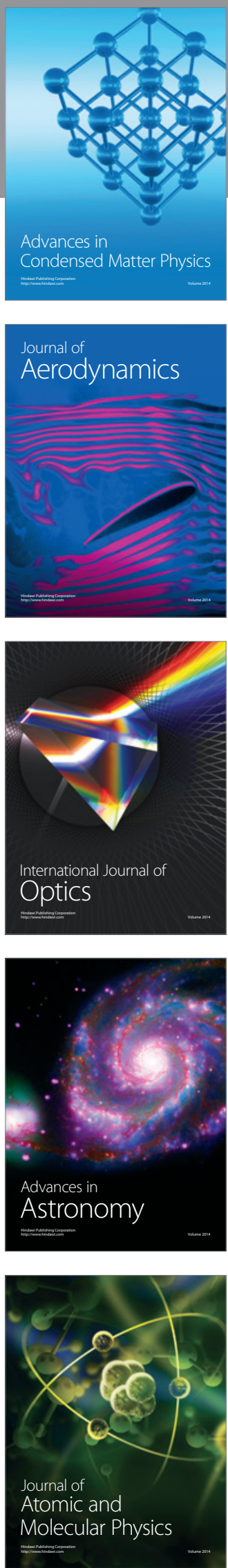